\title{
Mori-Tanaka Models for the Thermal \\ Conductivity of Composites with Interfacial \\ Resistance and Particle Size Distributions
}

\author{
Helmut J. Böhm ${ }^{\text {a,* }}$ Sergio Nogales ${ }^{\text {a,b }}$ \\ ${ }^{\mathrm{a}}$ Institute of Lightweight Design and Structural Biomechanics, \\ Vienna University of Technology, Gußhausstr. 27-29, A-1040 Vienna, Austria \\ ${ }^{\mathrm{b}}$ Max Planck Institute for Plasma Physics, \\ Boltzmannstr. 2, D-85748 Garching, Germany
}

\begin{abstract}
A Mori-Tanaka scheme is presented for modeling the overall thermal conduction behavior of composites containing reinforcements with interfacial resistances and prescribed size distributions. The approach is used for studying composites reinforced by spherical particles with monomodal and bimodal log-normal volume fraction distributions. Relevant effects of the distribution functions on the overall conductivity of the composites are found for particle volume fraction distributions with spans in excess of unity.
\end{abstract}

Key words: A. Metal-matrix composites, B. Modeling, B. Thermal properties, Particle size distributions

PACS: $16.60-\mathrm{Rc}, 81.05-\mathrm{Ni}$ 


\section{Introduction}

It is well known that interfacial resistances between reinforcements and matrix give rise to a size effect in the overall thermal conductivity of composites, with even highly conductive particles or fibers failing to increase the overall conductivity when their size falls below certain limits.

For composites reinforced by aligned continuous fibers or by spherical particles that show thermal interfacial resistances the dependence of the effective conductivity on the reinforcement size can be described by the well-known Maxwell effective medium method of Hasselman and Johnson [1]. Benveniste [2] obtained equivalent results by a Generalized Self-Consistent scheme and by a Mori-Tanaka theory. Mean field estimates of the above types can be interpreted in terms of replacing the actual particles or fibers, characterized by a given conductivity tensor and interfacial conductance, with fictitious inhomogeneities of the same shape that combine a suitably reduced "replacement conductivity" with a perfect interface. This replacement conductivity is chosen such that the resulting conductive behavior is equal to that of the actual configuration. Because the above approaches typically prescribe the same replacement conductivity for all particles or fibers they effectively treat all reinforcements as having the same size, without regard for the microgeometry underlying the chosen mean field model. As a consequence, these models pertain to composites containing reinforcements of equal size. Related approaches were developed for materials in which the reinforcements have a weakly conducting coating (or interphase) of finite thickness, see e.g. [3].

* Corresponding author, Tel.: +43 158801 31712, Fax.: +43 15880131799.

Email address: hjb@ilsb.tuwien.ac.at (Helmut J. Böhm).

URL: http://www.ilsb.tuwien.ac.at (Helmut J. Böhm). 
For spherical particles of isotropic conductivity $K^{(\mathrm{i})}$, interfacial conductance $h$, and diameter $d$ the replacement conductivity $K^{(\mathrm{i}, \mathrm{r})}$ takes the form [4]

$$
K^{(\mathrm{i}, \mathrm{r})}=K^{(\mathrm{i})} \frac{d h}{d h+2 K^{(\mathrm{i})}}
$$

When such "replacement particles" are embedded in a matrix of isotropic conductivity $K^{(\mathrm{m})}<K^{(\mathrm{i})}$ there exists a critical diameter $d_{\mathrm{c}}$, for which the replacement conductivity of the particle equals the conductivity of the matrix. This critical diameter can be evaluated from eqn.(1) as

$$
d_{\mathrm{c}}=\frac{2}{h} \frac{K^{(\mathrm{i})} K^{(\mathrm{m})}}{K^{(\mathrm{i})}-K^{(\mathrm{m})}}
$$

Particles with $d \leq d_{\mathrm{c}}$, accordingly, cannot lead to improvements of the overall conductivity of the composite.

A more accurate model for describing the overall conductive behavior of composites with interfacial thermal contact resistances was developed by Torquato and Rintoul [5] within the framework of three-point bounds. In this approach a three-point microstructural parameter is used to describe the statistics of the phase geometry, which, accordingly, explicitly enters the scheme. Expressions for three-point microstructural parameters as functions of the reinforcement volume fraction are available for a number of microgeometrical configurations [6]. For more general phase geometries, e.g. randomly dispersed particles that follow a given size distribution, appropriate microstructural parameters must be evaluated, which can be a complex task.

A further approach to modeling the overall conductivities of composites with interfacial resistances employs numerical engineering methods such as the Finite Element Method for evaluating the thermal fields in discrete microgeome- 
tries described, e.g., by unit cells. Such methods can handle a wide range of particle shapes, sizes, and arrangements, but are often expensive in terms of computational resources and analyst's time, realistic microgeometries tending to be challenging to mesh and giving rise to models with high numbers of degrees of freedom. Alternatively, numerical methods can be used to obtain replacement conductivities of individual particles or fibers, which are then introduced into mean field models. For a comparison between these two modeling strategies see e.g. [7].

To the authors' knowledge no low-cost method has been published that provides estimates for the overall conduction behavior of composites reinforced by particles that have a given interfacial resistance and follow prescribed size distributions. The present work is aimed at providing such a model on the basis of a Mori-Tanaka theory.

\section{Mori-Tanaka Approximations}

Mean field theories for describing the behavior of composite materials are typically formulated in terms of phase concentration tensors that connect phase averaged fields in reinforcements or matrix with the corresponding macroscopic fields. The concentration tensors relevant for thermal conduction prob-

lems are the gradient concentration tensors $\overline{\mathbf{A}}^{(\mathrm{p})}$ and the flux concentration tensors $\overline{\mathbf{B}}^{(\mathrm{p})}$ which are defined by the relationships

$$
\overline{\mathbf{d}}^{(\mathrm{p})}=\overline{\mathbf{A}}^{(\mathrm{p})}\langle\mathbf{d}\rangle \quad \text { and } \quad \overline{\mathbf{q}}^{(\mathrm{p})}=\overline{\mathbf{B}}^{(\mathrm{p})}\langle\mathbf{q}\rangle
$$


Here $\mathbf{d}$ and $\mathbf{q}$ stand for the thermal gradient and flux vectors, respectively, the superscript (p) indicates the phase (matrix or reinforcement), the angle brackets denote macroscopic fields, and overbars mark phase averaging.

The Mori-Tanaka estimates developed by Benveniste [8] for describing the effective elastic behavior of inhomogeneous materials can be directly extended to diffusion problems. In such an approach for thermal conduction the MoriTanaka gradient concentration tensors of matrix and non-dilute reinforcements, $\overline{\mathbf{A}}_{\mathrm{MT}}^{(\mathrm{m})}$ and $\overline{\mathbf{A}}_{\mathrm{MT}}^{(\mathrm{i})}$, respectively, take the form

$$
\begin{aligned}
\overline{\mathbf{A}}_{\mathrm{MT}}^{(\mathrm{m})} & =\left(\xi^{(\mathrm{m})} \mathbf{I}+\xi^{(\mathrm{i})} \overline{\mathbf{A}}_{\mathrm{dil}}^{(\mathrm{i})}\right)^{-1} \\
\overline{\mathbf{A}}_{\mathrm{MT}}^{(\mathrm{i})} & =\overline{\mathbf{A}}_{\mathrm{dil}}^{(\mathrm{i})}\left(\xi^{(\mathrm{m})} \mathbf{I}+\xi^{(\mathrm{i})} \overline{\mathbf{A}}_{\mathrm{dil}}^{(\mathrm{i})}\right)^{-1} .
\end{aligned}
$$

where all tensors are of rank 2. I denotes the identity tensor, $\xi^{(\mathrm{i})}$ stands for the reinforcement volume fraction, and $\xi^{(\mathrm{m})}=1-\xi^{(\mathrm{i})}$ is the matrix volume fraction. The gradient concentration tensor of dilute reinforcements with perfect interfaces, $\overline{\mathbf{A}}_{\text {dil }}^{(\mathrm{i})}$, can be written in analogy to Hill's [9] expressions for mechanical concentration tensors as

$$
\overline{\mathbf{A}}_{\text {dil }}^{(\mathrm{i})}=\left[\mathbf{I}+\mathbf{S R}^{(\mathrm{m})}\left(\mathbf{K}^{(\mathrm{i})}-\mathbf{K}^{(\mathrm{m})}\right)\right]^{-1},
$$

where $\mathbf{K}^{(\mathrm{i})}$ is the conductivity tensor of the reinforcements, $\mathbf{K}^{(\mathrm{m})}$ and $\mathbf{R}^{(\mathrm{m})}=$ $\left(\mathbf{K}^{(m)}\right)^{-1}$ are the conductivity and resistivity tensors, respectively, of the matrix, and $\mathbf{S}$ is the Eshelby tensor of the diffusion problem. Explicit expressions for $\mathbf{S}$ for ellipsoidal inclusions embedded in an isotropic matrix were given e.g. by Hatta and Taya [10]. Using the above tensors Mori-Tanaka estimates for the overall conductivity tensors $\mathbf{K}^{*}$ of two-phase composites can be obtained 


$$
\mathbf{K}^{*}=\mathbf{K}^{(\mathrm{m})}+\xi^{(\mathrm{i})}\left(\mathbf{K}^{(\mathrm{i})}-\mathbf{K}^{(\mathrm{m})}\right) \overline{\mathbf{A}}_{\mathrm{MT}}^{(\mathrm{i})}
$$

Equations (4) to (6) hold for general material symmetries of particles and matrix, pertain to aligned reinforcements with perfect interfaces, and do not show an intrinsic length scale. For spherical particles of isotropic conductivity that are embedded in an isotropic matrix the above equations reduce to the scalar Mori-Tanaka expressions given e.g. in [11].

Following Duschlbauer [12] the above Mori-Tanaka scheme can be extended to handle interfacial thermal resistances by introducing dilute replacement reinforcement conductivity tensors $\mathbf{K}^{(\mathrm{i}, \mathrm{r})}$ and the associated dilute replacement gradient concentration tensors, $\overline{\mathbf{A}}_{\text {dil }}^{(\mathrm{i}, \mathrm{r})}$, which must fulfill the consistency condition

$$
\overline{\mathbf{A}}_{\text {dil }}^{(\mathrm{i}, \mathrm{r})}=\frac{1}{\xi^{(\mathrm{i})}}\left(\mathbf{K}^{(\mathrm{i}, \mathrm{r})}-\mathbf{K}^{(\mathrm{m})}\right)^{-1}\left(\mathbf{K}_{\mathrm{dil}}^{*}-\mathbf{K}^{(\mathrm{m})}\right)
$$

The replacement tensors $\mathbf{K}^{(\mathrm{i}, \mathrm{r})}$ and $\overline{\mathbf{A}}_{\text {dil }}^{(\mathrm{i}, \mathrm{r})}$ are inserted into eqns.(4) and (6) in lieu of $\mathbf{K}^{(\mathrm{i})}$ and $\overline{\mathbf{A}}_{\text {dil }}^{(\mathrm{i})}$, respectively. Both replacement tensors depend on the size of the reinforcements and introduce an absolute length scale into the composite's overall conduction behavior.

For general distributions of the conductances on the interface, for general shapes of the reinforcements, and for general material symmetries of the constituents numerical methods must be used for obtaining $\mathbf{K}_{\text {dil }}^{*}, \mathbf{K}^{(\mathrm{i}, \mathrm{r})}$, and/or $\overline{\mathbf{A}}_{\mathrm{dil}}^{(\mathrm{i}, \mathrm{r})}$. For this purpose dilute models consisting of single reinforcements embedded in large matrix regions may be employed, which are subjected to three linearly independent applied temperature gradients. $\overline{\mathbf{A}}_{\text {dil }}^{(\mathrm{i}, \mathrm{r})}$ can then be evalu- 
ated from the phase averaged fields in the inhomogeneity and $\mathbf{K}_{\text {dil }}^{*}$ from averages over the whole model, compare [12], with $\mathbf{K}^{(\mathrm{i}, \mathrm{r})}$ following from eqn.(7).

If the reinforcements are of ellipsoidal shape, the phase conductivities are isotropic and the interfacial conductances are distributed confocally with the particle shape (implying homogeneous conductances for spherical particles and aligned continuous cylindrical fibers, and inhomogeneous ones otherwise), analytical expressions for the replacement connectivities can be obtained $[12,13]$ and the dilute replacement gradient concentration tensors can be evaluated from them as

$$
\overline{\mathbf{A}}_{\mathrm{dil}}^{(\mathrm{i}, \mathrm{r})}=\left[\mathbf{I}+\mathbf{S R}^{(\mathrm{m})}\left(\mathbf{K}^{(\mathrm{i}, \mathrm{r})}-\mathbf{K}^{(\mathrm{m})}\right)\right]^{-1}
$$

in analogy to eqn.(5). For the special case of spherical reinforcements with homogeneous interfacial conductances the replacement conductivity tensor is isotropic and its only nonzero components are the diagonals, which are given by eqn.(1). The pertinent Eshelby tensor has the same structure with $S_{k k}=1 / 3[10]$, so that a Mori-Tanaka expression for the effective thermal conductivity of composites reinforced by spherical particles with finite interfacial conductance can be recovered from eqns.(1), (4), (6) and (8) as

$$
K^{*}=K^{(\mathrm{m})}+\frac{3 \xi^{(\mathrm{i})} K^{(\mathrm{m})}\left(K^{(\mathrm{i}, \mathrm{r})}-K^{(\mathrm{m})}\right)}{3 K^{(\mathrm{m})}+\xi^{(\mathrm{m})}\left(K^{(\mathrm{i}, \mathrm{r})}-K^{(\mathrm{m})}\right)}
$$

compare [2]. This relationship is equivalent to the Maxwell estimates of Hasselman and Johnson [1].

In order to account for prescribed size distributions of the reinforcements the Mori-Tanaka expressions (4) and (7) can be extended to multiple reinforcement phases $(j)$, each of which corresponds to reinforcements of a given size 
class described by appropriate replacement tensors $\mathbf{K}^{(\mathrm{j}, \mathrm{r})}$ and $\overline{\mathbf{A}}_{\mathrm{dil}}^{(\mathrm{j}, \mathrm{r})}$. In such a scheme the Mori-Tanaka reinforcement gradient concentration tensors pertaining to $N$ size classes of reinforcements with volume fractions $\xi^{(\mathrm{j})}$, take the form

$$
\overline{\mathbf{A}}_{\mathrm{MT}}^{(\mathrm{i}, \mathrm{r})}=\overline{\mathbf{A}}_{\mathrm{dil}}^{(\mathrm{i}, \mathrm{r})}\left(\xi^{(\mathrm{m})} \mathbf{I}+\sum_{\mathrm{j}=1}^{N} \xi^{(\mathrm{j})} \overline{\mathbf{A}}_{\mathrm{dil}}^{(\mathrm{j}, \mathrm{r})}\right)^{-1}
$$

and the estimate for the macroscopic conductivity tensor of the composite follows as

$$
\mathbf{K}^{*}=\mathbf{K}^{(\mathrm{m})}+\sum_{\mathrm{j}=1}^{N} \xi^{(\mathrm{j})}\left(\mathbf{K}^{(\mathrm{j}, \mathrm{r})}-\mathbf{K}^{(\mathrm{m})}\right) \overline{\mathbf{A}}_{\mathrm{MT}}^{(\mathrm{j}, \mathrm{r})}
$$

The phase volume fractions fulfil the relationship $\xi^{(\mathrm{m})}+\sum_{\mathrm{j}=1}^{N} \xi^{(\mathrm{j})}=1$.

This modeling approach can also handle composites with interfacial resistances that depend on the reinforcement size, $h=h(d)$. If the reinforcements are nonspherical and not aligned, an orientation averaging scheme must be introduced at the level of the dilute replacement tensors in analogy to [7]. It is worth noting that the algorithm does not pose any restrictions on the width of the individual size classes.

There are two special cases in which the above tensor expressions can be reduced to scalar relationships in a straightforward way. For isotropic reinforcements of spherical shape that are embedded in an isotropic matrix, all the tensors involved in eqns.(10) and (11) are of diagonal form with identical diagonal elements, giving rise to the scalar relationship

$$
K^{*}=K^{(\mathrm{m})}+\frac{\sum_{\mathrm{j}=1}^{N} \xi^{(\mathrm{j})}\left(K^{(\mathrm{j}, \mathrm{r})}-K^{(\mathrm{m})}\right) A_{\mathrm{dil}}^{(\mathrm{j}, \mathrm{r})}}{\xi^{(\mathrm{m})}+\sum_{\mathrm{j}=1}^{N} \xi^{(\mathrm{j})} A_{\mathrm{dil}}^{(\mathrm{j}, \mathrm{r})}}
$$


with

$$
A_{\mathrm{dil}}^{(\mathrm{j}, \mathrm{r})}=\frac{3 K^{(\mathrm{m})}}{2 K^{(\mathrm{m})}+K^{(\mathrm{j}, \mathrm{r})}}
$$

where $K^{(\mathrm{j}, \mathrm{r})}$ is obtained by evaluating eqn.(1) with the particle diameter pertinent to size class $(\mathrm{j})$.

Equation (12) also holds for the transverse macroscopic conductivity of composites reinforced by unidirectional continuous fibers that show non-uniform diameters and interfacial resistances. The pertinent diagonal elements in the Eshelby tensor, however, take the value of $1 / 2$ and the corresponding transverse dilute gradient concentration factors for the fibers are obtained from eqn.(5) as

$$
A_{\mathrm{dil}, \mathrm{T}}^{(\mathrm{i}, \mathrm{r}}=\frac{2 K^{(\mathrm{m})}}{K^{(\mathrm{m})}+K^{(\mathrm{i}, \mathrm{r})}}
$$

The axial gradient concentration factor for such materials is unity, the overall axial conductivity follows the rule of mixtures, and no size effect due to the interface is present in the axial behavior.

An analogous Mori-Tanaka scheme for studying the thermal conduction properties of composites with aligned reinforcements having finite interfacial conductances can be formulated in terms of the replacement flux concentration tensors $\overline{\mathbf{B}}^{(\mathrm{i}, \mathrm{r})}$, compare eqn.(3), and resistivity tensors $\mathbf{R}^{(\mathrm{i}, \mathrm{r})}=\left(\mathbf{K}^{(\mathrm{i}, \mathrm{r})}\right)^{-1}$ of the reinforcements. In such a scheme the equivalent to eqn.(8) takes the form

$$
\overline{\mathbf{B}}_{\mathrm{dil}}^{(\mathrm{i}, \mathrm{r})}=\left[\mathbf{I}+\mathbf{K}^{(\mathrm{m})}\left(\mathbf{I}-\mathbf{S}^{(\mathrm{m})}\right)\left(\mathbf{R}^{(\mathrm{i}, \mathrm{r})}-\mathbf{R}^{(\mathrm{m})}\right)\right]^{-1} .
$$

All relations presented in the present section are explicit. Equations (10) to (14) are not subject to restrictions in the volume fraction distributions that 
can be handled. Using the tensorial relations, eqns.(10) and (11), for general reinforcement shapes and volume fraction distributions tends to be time consuming, a set of three numerical analyses with linearly independent thermal loads being required to evaluate the dilute replacement tensors for each size class of inhomogeneities considered. The scalar equations obtained for the special cases of isotropic spheres and transversally isotropic aligned continuous fibers with homogeneous interfacial conductances, however, can efficiently handle a high number of size classes. Accordingly, spherical reinforcements were chosen for the following examples.

\section{Examples and Discussion}

In this section the Mori-Tanaka expressions for composites reinforced by equiaxed particles that show an interfacial resistance and follow prescribed size distributions, eqns.(12) and (13), are applied to a model composite, the material parameters of which are given in table 1 . This model material approximates diamond-aluminum composites, which were reported by Kleiner et al. [14] to reach effective conductivities of up to $670 \mathrm{~W} / \mathrm{mK}$ at particle volume fractions of $\xi \approx 0.6$. The conduction contrast between particles and matrix evaluates as $K^{(\mathrm{i})} / K^{(\mathrm{m})}=7.5$ and the critical particle diameter takes a value of $d_{\mathrm{c}}=10^{-5} \mathrm{~m}$.

Figure 1 shows predictions for the dependence of the overall conductivity on the particle diameter for spherical particles of equal size. The curve "E-MTM" pertains to the Mori-Tanaka estimates, eqn.(9), which, as noted before, are identical to the predictions of the Hasselman-Johnson [1] model and of Benveniste's [2] self-consistent scheme. The curves "T-R LB" and "T-R UB" give 
the lower and upper three-point bounds of Torquato and Rintoul [5] evaluated with the expressions of Miller and Torquato [17] for the three-point microstructural parameter pertaining to randomly positioned hard spheres of equal size. Predictions that combine the differential scheme [15] with eqn. (1) in the spirit of [16] are marked as "DS". All four curves show a marked dependence of the overall thermophysical behavior on the particle size, with the predicted overall conductivities decreasing by nearly a factor of ten within three orders of magnitude of the diameters. For large particle diameters the interfacial effects vanish and the Mori-Tanaka estimates merge into the lower Hashin-Shtrikman bounds [18] for the conductivity of isotropic composites reinforced by particles with perfect interfaces. The Torquato-Rintoul bounds become equal to the three-point bounds [6] for spheres with ideal interfacial conductances, which are much tighter than the Hashin-Shtrikman bounds, so that the Mori-Tanaka estimates lie a few percent below the three-point bounds in this regime. For the present material parameters (but not for high phase contrasts) the predictions of the differential scheme slightly exceed the upper three-point bounds in the large particle limit. For $d \ll d_{\mathrm{c}}$ the interfacial resistances make the reinforcements behave like non-conducting inhomogeneities and the Mori-Tanaka estimates approach the corresponding Hashin-Shtrikman upper bounds. Like the predictions of the differential scheme they fall within the Torquato-Rintoul bounds in the small particle regime. At the critical diameter $d_{c}$, where the composite thermally behaves like a homogeneous material, all the above solutions coincide with the conductivity of the unreinforced matrix.

For studying the effects of non-uniformly sized particles a monomodal lognormal distribution of the particle volume fractions in terms of the particle 
diameters,

$$
\xi(d)=\frac{\exp \left(-\left[\frac{\ln (d)-\mu}{\sqrt{2} \sigma}\right]^{2}\right)}{\sqrt{2 \pi} d \sigma},
$$

was prescribed. Such log-normal distribution functions can give reasonable approximations to the size distribution of particles in MMCs, see e.g. Molina et al. [19]. The two parameters $\mu$ and $\sigma$ in eqn.(16) can be easily evaluated from standard descriptors of probability densities, such as the expected value, the standard deviation, the median $M=D(50)$, and the span $S=[D(90)-$ $D(10)] / D(50)$ of the particle diameters. Here $D(x)$ stands for the diameter below which $x \%$ of the particle diameters are found. In the present study results are parameterized in terms of medians and spans of the distributions, for which the relations

$$
\begin{aligned}
& \mu=\ln (M) \\
& \sigma=\frac{1}{1.2816} \ln \left(\frac{S+\sqrt{S^{2}+4}}{2}\right) .
\end{aligned}
$$

hold. For use with eqn.(12) the smooth distributions (16) were discretized into size classes, 1000 logarithmically spaced classes being typically employed per decade of particle diameters.

The dependence of the overall conductivities of the composite on the median diameters of spherical particles with monomodal log-normal size distributions is shown in figure 2 for three values of the span. The predictions for a span of unity, marked as D-MTM/L,S=1, hardly deviate from the Mori-Tanaka solution for particles of equal size, curve E-MTM. Spans of 5 and 9 do not qualitatively change the conductivity vs. size curves, but make the transition between the large and small diameter regimes more gradual, i.e., there is a decrease of the overall conductivity in the transition region for median di- 
ameters above $d_{\mathrm{c}}$ and some increase for median diameters below this value. For wide distributions with $S=9$ the predicted reductions in $K^{*}$ can approach $20 \%$ of the value pertaining to equally sized particles for the system considered. Figure 3 depicts the dependence of the overall conductivities of composites reinforced by spherical particles of log-normal size distribution for a wide range of medians and spans. It confirms that log-normal size distributions with spans below unity do not give rise to noticeable effects on the macroscopic thermophysical behavior of composites with a moderate thermal conductivity contrast between particles and matrix.

A somewhat larger sensitivity to the span is found for "cropped" uniform monomodal distributions of the volume fractions,

$$
\xi(d)= \begin{cases}\frac{0.8}{M S} & \text { for } \quad M(1-0.625 S) \leq d \leq M(1+0.625 S) \\ 0 & \text { otherwise }\end{cases}
$$

for which the span cannot exceed a value of 1.6 and the maximum particle diameter is limited to twice the median diameter. As can be seen in figure 4 volume fractions of this type lead to a qualitatively different behavior than lognormal ones. For wide cropped uniform volume fraction distributions (curve $\mathrm{D}-\mathrm{MT} / \mathrm{U}, \mathrm{S}=1.59)$ a decrease in the overall conductivity is predicted for all median diameters of spherical particles compared to composites reinforced with uniformly sized spheres (curve E-MTM).

In order to achieve high particle volume fractions in composites larger and smaller particles may be mixed, resulting in bimodal particle size distributions. For studying the thermal conduction behavior of such composites, systems containing two populations of log-normally distributed particles, referred 
to as "small" and "large" in the following, were modeled. The partial distribution functions of the two populations of particles are characterized by their medians, $M^{\mathrm{S}}$ and $M^{\mathrm{L}}$, and by their spans, $S^{\mathrm{S}}$ and $S^{\mathrm{L}}$, respectively. Particle mixes containing different contributions of the two populations are described by a "mixing parameter", $\Xi$, with $\Xi=0$ and $\Xi=1$ denoting monomodal distributions consisting only of the small or large particles, respectively. The total volume fraction of the particles is kept fixed at $\xi^{(i)}=0.6$.

Figures 5 and 6 present predictions of the effective conductivity as functions of $\Xi$ evaluated for log-normal partial distributions of equal span, $S=S^{\mathrm{S}}=S^{\mathrm{L}}$, and for median particle diameters that differ by a factor of 10. In addition, "rule of mixture" approximations obtained from the relation

$$
K_{\mathrm{ROM}}^{*}=\Xi K^{*}\left(d=M^{\mathrm{S}}\right)+(1-\Xi) K^{*}\left(d=M^{\mathrm{L}}\right)
$$

are shown, which are linear interpolations between results obtained with eqn.(9) for composites reinforced with particles having diameters equal to the median diameters of the small, $d=M^{\mathrm{S}}$, and large, $d=M^{\mathrm{L}}$, populations of particles, respectively. In cases where both median diameters are either much smaller or much larger than $d_{\mathrm{c}}$, the values of $K^{*}$ for both partial distributions hardly differ and eqn.(19) is a good approximation for spans less than unity.

For obtaining figure 5 the median diameters of the partial distributions were chosen as $M^{\mathrm{S}}=10^{-4} \mathrm{~m}$ and $M^{\mathrm{L}}=10^{-3} \mathrm{~m}$, i.e., they are close to the righthand "shoulder" of the overall conductivities as shown in figure 1. The rule of mixture approximation to the effective conductivity can be seen to nearly coincide with the Mori-Tanaka estimates for small to moderate spans of the partial distributions, $S \leq 1$, in this case. For very wide partials with spans of 
$S=10$ (where the two peaks of the bimodal distribution merge), the effective conductivities are markedly reduced for all values of $\Xi$, but a linear dependence on $\Xi$ is retained, i.e., the predictions of eqn.(12) and (13) closely follow a linear interpolation between the results pertaining to the corresponding monomodal volume fraction distributions.

Figure 6, in contrast, pertains to a situation where the median diameters straddle the critical diameter $d_{\mathrm{c}}, M^{\mathrm{S}}=3.1623 \times 10^{-6} \mathrm{~m}$ and $M^{\mathrm{L}}=3.1623 \times 10^{-5} \mathrm{~m}$. Due to the smaller particle sizes the predicted overall conductivities are generally much lower than in figure 5. The Mori-Tanaka estimates for the overall conductivity at low to moderate spans, $S \leq 1$, in this case are obviously nonlinear in $\Xi$ and they are clearly distinct from the ROM data. Wide partial distributions with $S=10$ again lead to a different overall behavior, the nonlinearity of the curves being somewhat smaller. The nonlinearity of the $K^{*}$ vs. $\Xi$ relationship, of course, vanishes as the ratio between median diameters of the partial distribution functions, $M^{\mathrm{L}} / M^{\mathrm{S}}$, approaches unity, where the two partials merge.

Predictions pertaining to monomodal log-normal particle volume fraction distributions with $M=M^{\mathrm{L}}, S=1$ and $\xi^{(\mathrm{i})}=0.3$ are also plotted in figures 5 and 6 for comparison. As expected, the use of bimodally sized particles provide considerable improvements in the overall conductivity if the median diameters of both populations are sufficiently large, compare figure 5. Adding particles that are smaller than the critical size $d_{\mathrm{c}}$, in contrast, while increasing the particle volume fraction, produces limited gains at best and may actually degrade the macroscopic conductivity of the composite, see figure 6 .

Finally, it is worth noting that difficulties analogous to the ones reported for 
extended Mori-Tanaka models of the overall thermoelastic response of composites, viz. the prediction of non-symmetric "elastic tensors" [20,21] have not been observed in modeling the conduction behavior, even in cases where the reinforcements are both nonaligned and anisotropic and the resulting overall behavior is anisotropic, too [7].

\section{Conclusions}

The proposed Mori-Tanaka scheme provides a flexible tool for modeling the overall thermal conductivity of composites reinforced by non-uniformly sized particles or fibers with interfacial resistances. It is especially simple in describing macroscopically isotropic materials that contain spherical particles following prescribed size distributions. The model underestimates the overall conductivities for large particles and may tend to overestimate them for small inhomogeneities, the inaccuracies being minor for moderate conductivity contrasts between reinforcements and matrix. The method is suitable for handling general distributions of the reinforcement volume fractions given as functions of the particle diameter.

Wide monomodal volume fraction distribution functions, with spans considerably exceeding unity, are predicted to lead to noticeable differences in the overall conduction response compared to particles of uniform size. For narrow distributions, in contrast, these effects are negligible and simpler methods, such as the Hasselman--Johnson estimates, give comparable results. Bimodal volume fraction distributions can give rise to nontrivial behavior when the partials are sufficiently far apart and straddle the critical diameter, whereas straightforward interpolation can give acceptable results in other cases. 


\section{Acknowledgments}

This work was carried out within the framework of the European Integrated Project "ExtreMat" (contract NMP-CT-2004-500253) with financial support from the European Community. It only reflects the views of the authors and the European Community is not liable from any use of the information contained therein.

\section{References}

[1] D. Hasselman, L. Johnson, Effective thermal conductivity of composites with interfacial thermal barrier resistance, J. Compos. Mater. 21 (1987) 508-515.

[2] Y. Benveniste, Effective thermal conductivity of composites with a thermal contact resistance between the constituents. Non-dilute case, J. Appl. Phys. 61 (1987) 2840-2843.

[3] M. Dunn, M. Taya, The effective thermal conductivity of composites with coated reinforcements and the application to imperfect interfaces, J. Appl. Phys. 73 (1993) 1711-1722.

[4] Y. Benveniste, T. Miloh, The effective conductivity of composites with imperfect thermal contact at constituent interfaces, Int. J. Engng. Sci. 24 (1986) 15371552.

[5] S. Torquato, D. Rintoul, Effect of the interface on the properties of composite media, Phys. Rev. Lett. 75 (1995) 4067-4070.

[6] S. Torquato, Random Heterogeneous Media, Springer-Verlag, New York, NY, 2002. 
[7] D. Duschlbauer, H. Böhm, H. Pettermann, Computational simulation of composites reinforced by planar random fibers: Homogenization and localization by unit cell and mean field approaches, J. Compos. Mater. 40 (2006) 2217-2234.

[8] Y. Benveniste, A new approach to the application of Mori-Tanaka's Theory in composite materials, Mech. Mater. 6 (1987) 147-157.

[9] R. Hill, A self-consistent mechanics of composite materials, J. Mech. Phys. Sol. 13 (1965) 213-222.

[10] H. Hatta, M. Taya, Equivalent inclusion method for steady state heat conduction in composites, Int. J. Engng. Sci. 24 (1986) 1159-1172.

[11] Y. Benveniste, On the effective thermal conductivity of multiphase composites, J. Appl. Math. Phys. 37 (1986) 696-713.

[12] D. Duschlbauer, Computational Simulation of the Thermal Conductivity of MMCs under Consideration of the Inclusion-Matrix Interface, VDI-Verlag, Düsseldorf, Germany, 2004.

[13] D. Duschlbauer, H.E. Pettermann, H.J. Böhm, Heat conduction of a spheroidal inhomogeneity with imperfectly bonded interface, J. Appl. Phys. 94 (2003) 1539-1549.

[14] S. Kleiner, F. Khalid, P. Ruch, S. Meier, O. Beffort, Effect of diamond crystallographic orientation on dissolution and carbide formation in contact with liquid aluminium, Scr. mater. 55 (2006) 291-294.

[15] D.A.G. Bruggemann, Berechnung verschiedener physikalischer Konstanten von heterogenen Substanzen. I. Dielektrizitätskonstanten und Leitfähigkeiten der Mischkörper aus isotropen Substanzen; Ann. Phys. 24 (1935) 636-679.

[16] J. Molina, J. Narciso, L. Weber, A. Mortensen, E. Louis, Thermal conductivity of $\mathrm{Al}-\mathrm{SiC}$ composites with monomodal and bimodal particle size distribution; submitted to Mater. Sci. Engng. A. 
[17] C. Miller, S. Torquato, Effective conductivity of hard sphere suspensions, J. Appl. Phys. 68 (1990) 5486-5493.

[18] Z. Hashin, S. Shtrikman, A variational approach to the theory of the effective magnetic permeability of multiphase materials, J. Appl. Phys. 33 (1962), 31253131.

[19] J. Molina, E. Piñero, J. Narciso, C. García-Cordovilla, E. Louis, Liquid metal infiltration into ceramic particle preforms with bimodal size distributions, Curr. Opin. Sol. State Mater. Sci. 9 (2006) 202-210.

[20] Y. Benveniste, G. Dvorak, T. Chen, On diagonal and elastic symmetry of the approximate effective stiffness tensor of heterogeneous media, J. Mech. Phys. Sol. 39 (1991) 927-946.

[21] M. Ferrari, Asymmetry and the high concentration limit of the Mori-Tanaka effective medium theory, Mech. Mater. 11 (1991) 251-256. 


\section{Figure Captions}

Figure 1: Predictions for the size dependent effective conductivities of composites reinforced by spherical particles of equal size (volume fraction $\xi^{(\mathrm{i})}=0.6$, material parameters following table 6) obtained by a Mori-Tanaka scheme (EMTM), a differential scheme (DS), as well as the three-point lower (T-R LB) and upper (T-R UB) bounds of Torquato and Rintoul [5].

Figure 2: Mori-Tanaka predictions for the size dependent effective conductivities of composites reinforced by particles (volume fraction $\xi^{(\mathrm{i})}=0.6$ ) of equal size (E-MTM) and by particles having log-normal size distributions (DMTM/L) with spans of $S=1, S=5$ and $S=9$.

Figure 3: Mori-Tanaka predictions for the effective conductivities of composites reinforced by particles (volume fraction $\xi^{(\mathrm{i})}=0.6$ ) with monomodal lognormal size distributions described by the medians and spans of the particle diameters.

Figure 4: Mori-Tanaka predictions for the size dependent effective conductivities of composites reinforced by particles (volume fraction $\xi^{(i)}=0.6$ ) of equal size (E-MTM) and by particles having a uniform size distribution with a span of $\mathrm{S}=1.59$ (D-MTM/U).

Figure 5: Comparison of "rule of mixture" (ROM) and Mori-Tanaka predictions (B-MTM) for the conductivities of composites reinforced by particles having a bimodal size distribution with $M^{\mathrm{S}}=10^{-4} \mathrm{~m}$ and $M^{\mathrm{L}}=10^{-3} \mathrm{~m}$ given as functions of the mixing parameter $\Xi$ and parameterized by the span 
$S=S^{\mathrm{S}}=S^{\mathrm{L}}$ of the two log-normal partial distributions. The result for a monomodal log-normal size distribution with $\xi=0.3, M=1.0 \times 10^{-3} \mathrm{~m}$ and $S=1$ is marked as D-MTM/L,0.3.

Figure 6: Comparison of "rule of mixture" (ROM) and Mori-Tanaka predictions (B-MTM) for the conductivities of composites reinforced by particles having a bimodal size distribution with $M^{\mathrm{S}}=3.1623 \times 10^{-6} \mathrm{~m}$ and $M^{\mathrm{L}}=3.1623 \times$ $10^{-5} \mathrm{~m}$ given as functions of the mixing parameter $\Xi$ and parameterized by the span $S=S^{\mathrm{S}}=S^{\mathrm{L}}$ of the two log-normal partial distributions. The result for a monomodal log-normal size distribution with $\xi=0.3, M=3.1623 \times 10^{-5} \mathrm{~m}$ and $S=1$ is marked as D-MTM/L,0.3. 


\section{Tables}

Table 1

Material parameters of the model material employed in generating figures 1 to 6 .

\begin{tabular}{cccc}
\hline$K^{(\mathrm{i})}$ & $K^{(\mathrm{m})}$ & $h$ & $\xi^{(\mathrm{i})}$ \\
{$[\mathrm{W} / \mathrm{mK}]$} & {$[\mathrm{W} / \mathrm{mK}]$} & {$\left[\mathrm{W} / \mathrm{m}^{2} \mathrm{~K}\right]$} & {$[1]$} \\
\hline 1800 & 240 & $5.538 \times 10^{7}$ & 0.6 \\
\hline
\end{tabular}




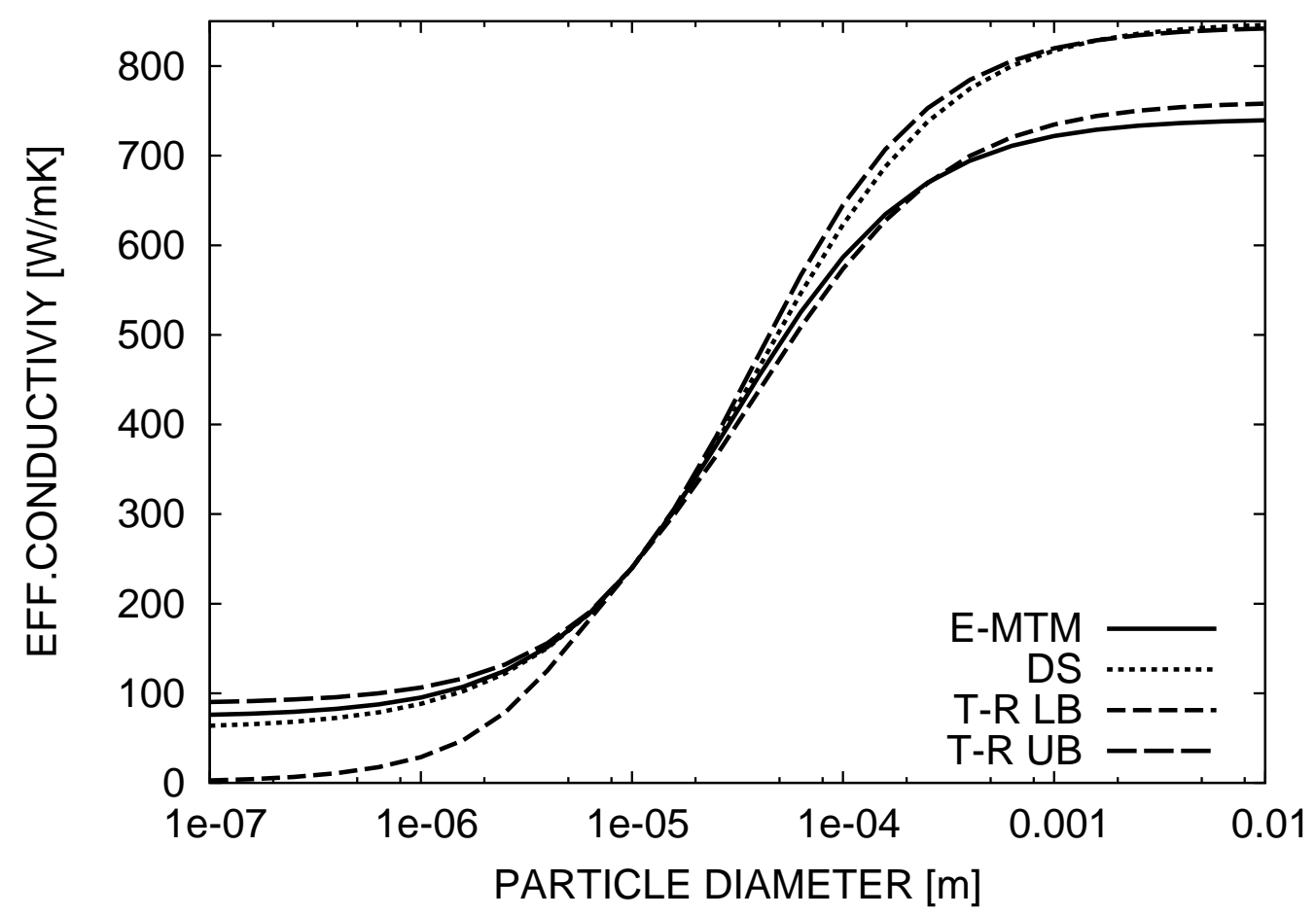

Fig. 1. Predictions for the size dependent effective conductivities of composites reinforced by spherical particles of equal size (volume fraction $\xi^{(\mathrm{i})}=0.6$, material parameters following table 6) obtained by a Mori-Tanaka scheme (E-MTM), a differential scheme (DS), as well as the three-point lower (T-R LB) and upper (T-R UB) bounds of Torquato and Rintoul [5]. 


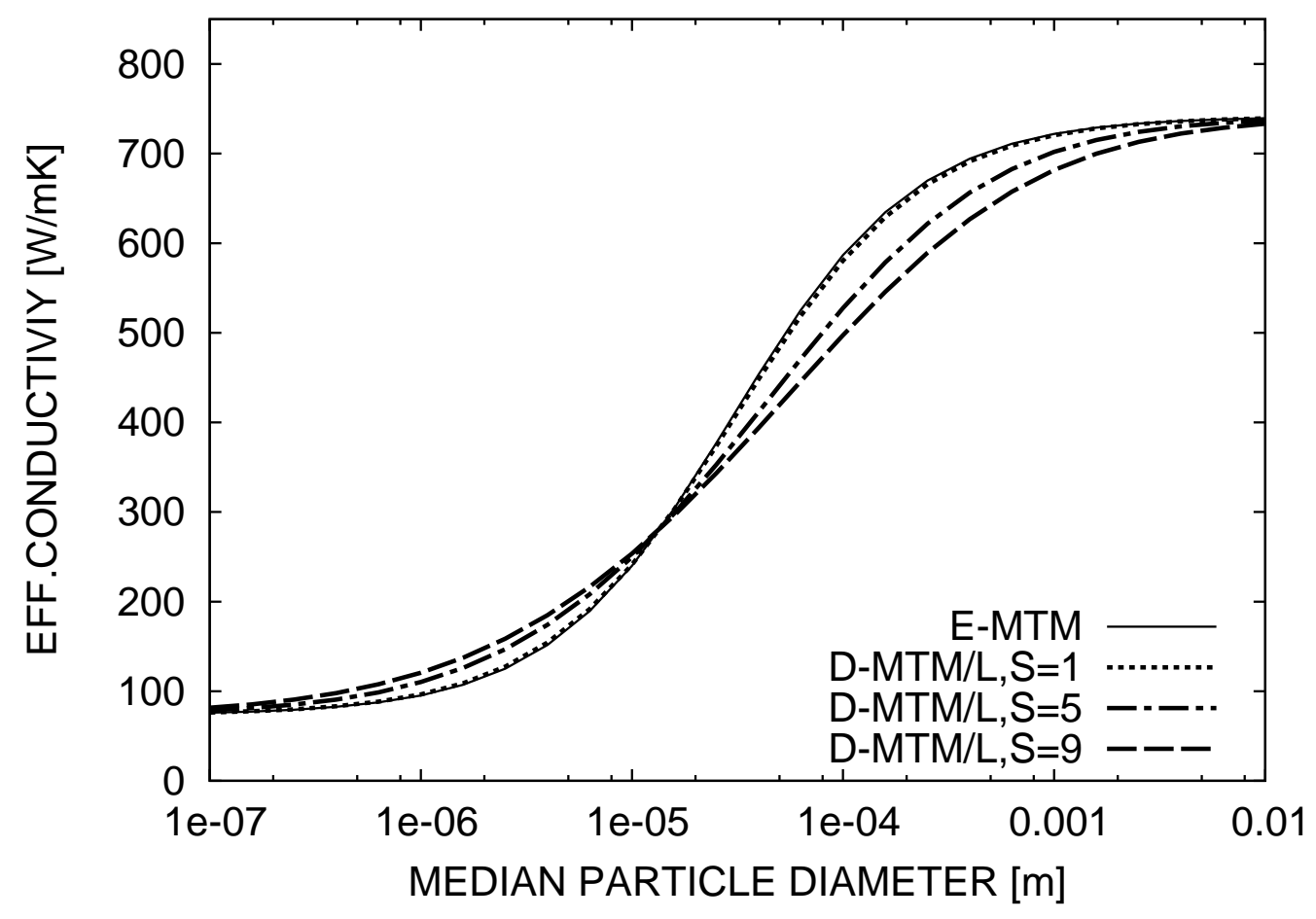

Fig. 2. Mori-Tanaka predictions for the size dependent effective conductivities of composites reinforced by particles (volume fraction $\left.\xi^{(\mathrm{i})}=0.6\right)$ of equal size $(\mathrm{E}-\mathrm{MTM})$ and by particles having log-normal size distributions (D-MTM/L) with spans of $S=1, S=5$ and $S=9$. 


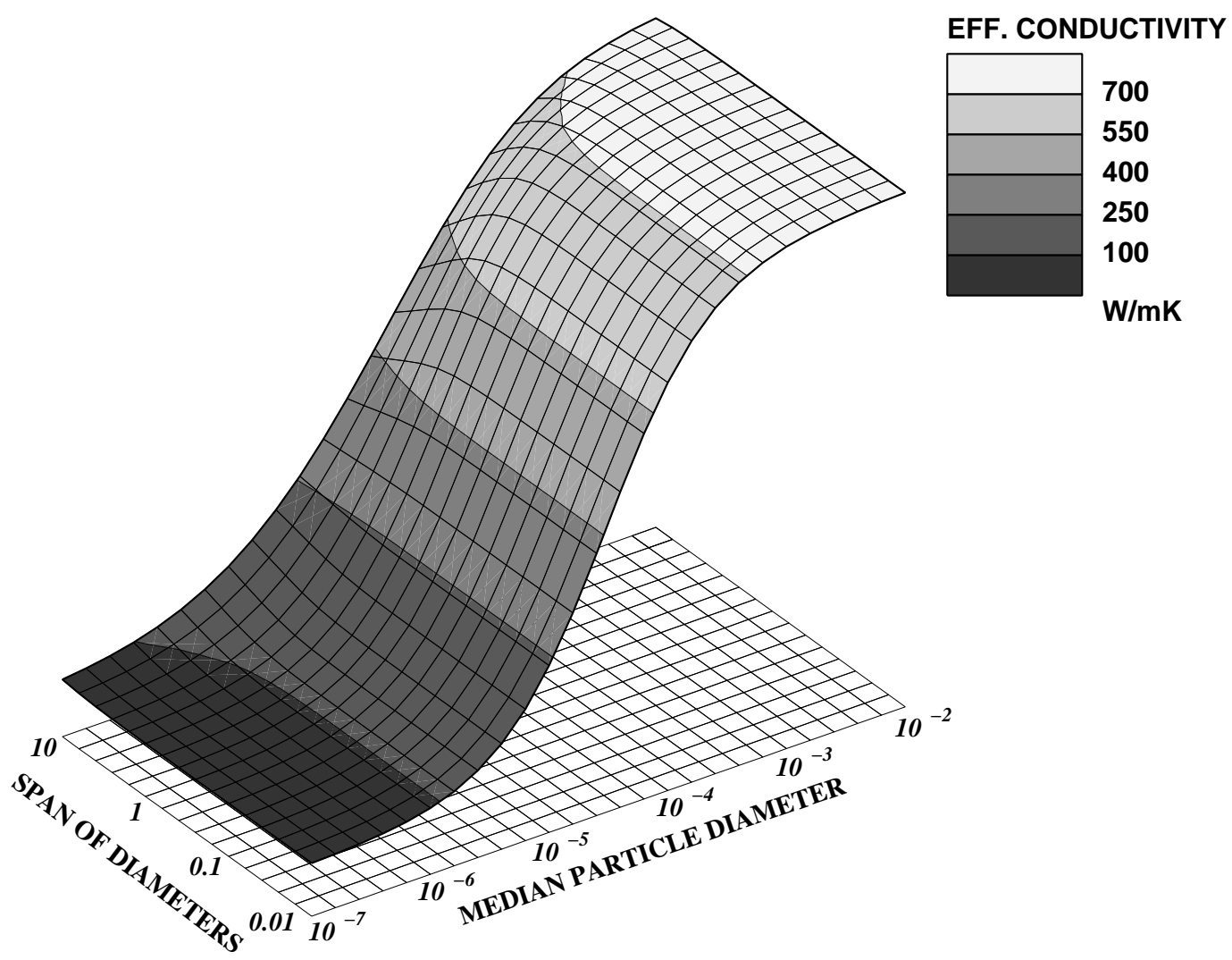

Fig. 3. Mori-Tanaka predictions for the effective conductivities of composites reinforced by particles (volume fraction $\xi^{(\mathrm{i})}=0.6$ ) with monomodal log-normal size distributions described by the medians and spans of the particle diameters. 


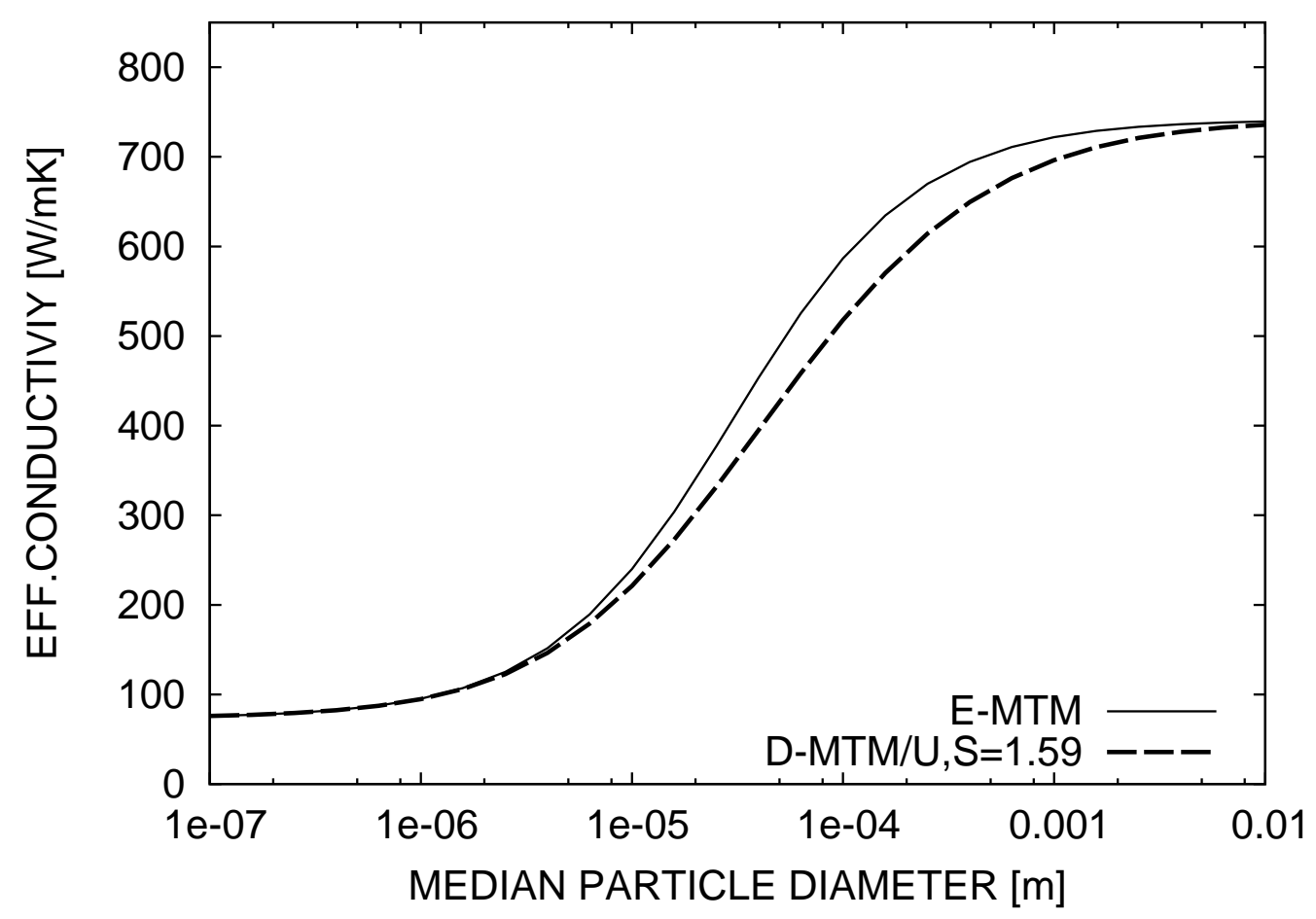

Fig. 4. Mori-Tanaka predictions for the size dependent effective conductivities of composites reinforced by particles (volume fraction $\xi^{(i)}=0.6$ ) of equal size (E-MTM) and by particles having a uniform size distribution with a span of $\mathrm{S}=1.59$ (D-MTM/U). 


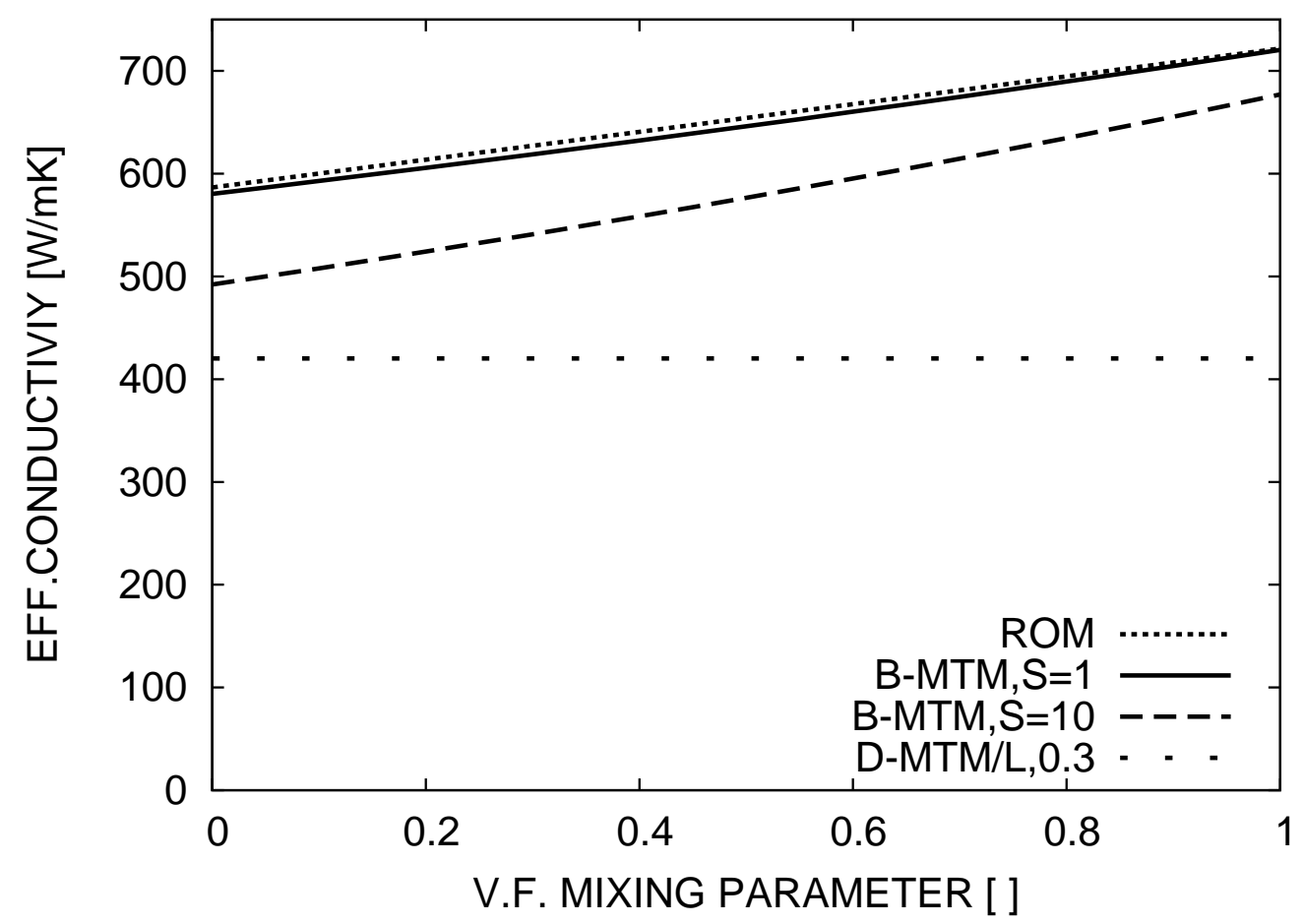

Fig. 5. Comparison of "rule of mixture" (ROM) and Mori-Tanaka predictions (B-MTM) for the conductivities of composites reinforced by particles having a bimodal size distribution with $M^{\mathrm{S}}=10^{-4} \mathrm{~m}$ and $M^{\mathrm{L}}=10^{-3} \mathrm{~m}$ given as functions of the mixing parameter $\Xi$ and parameterized by the span $S=S^{\mathrm{S}}=S^{\mathrm{L}}$ of the two log-normal partial distributions. The result for a monomodal log-normal size distribution with $\xi=0.3, M=1.0 \times 10^{-3} \mathrm{~m}$ and $S=1$ is marked as D-MTM/L,0.3. 


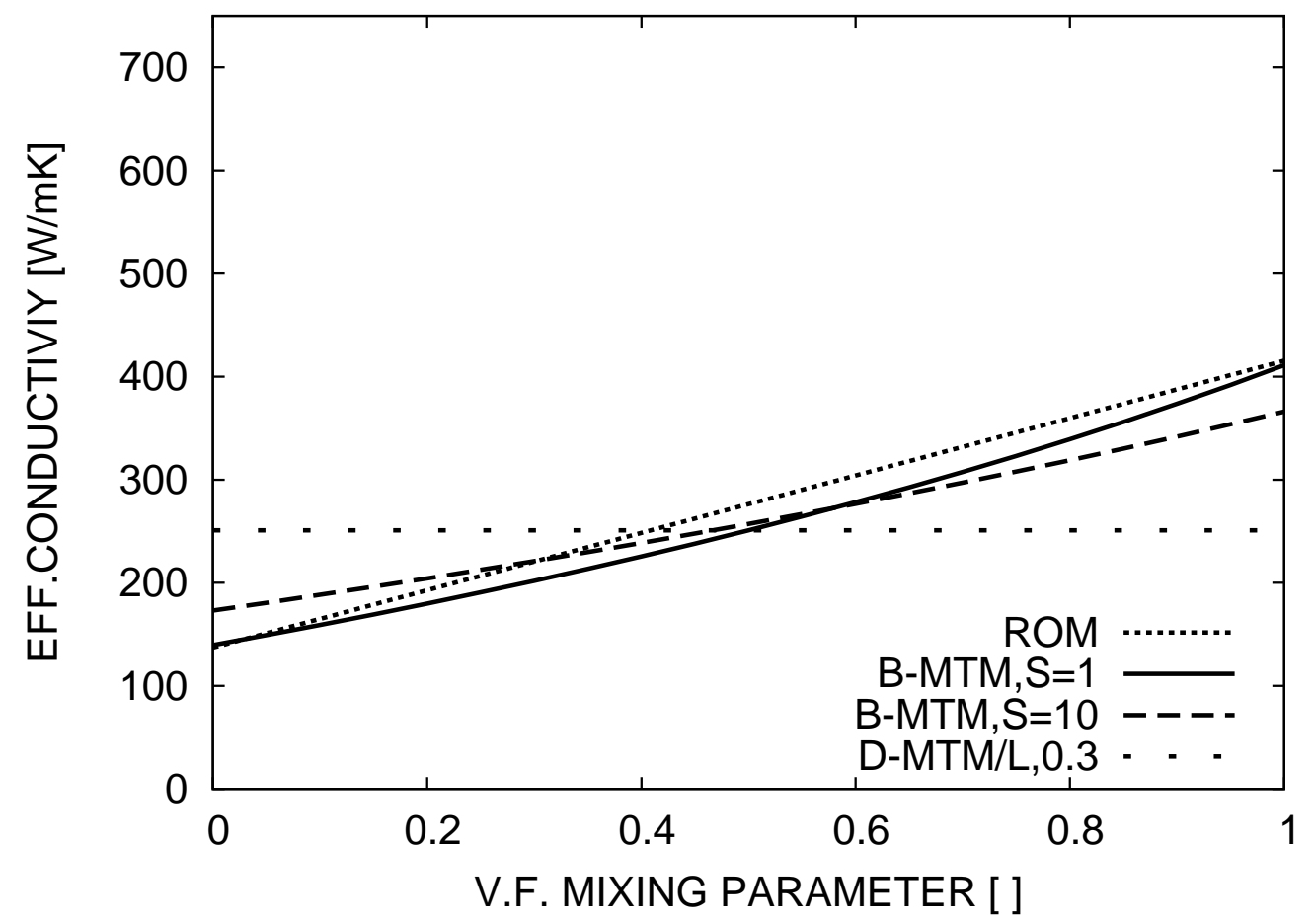

Fig. 6. Comparison of "rule of mixture" (ROM) and Mori-Tanaka predictions (B-MTM) for the conductivities of composites reinforced by particles having a bimodal size distribution with $M^{\mathrm{S}}=3.1623 \times 10^{-6} \mathrm{~m}$ and $M^{\mathrm{L}}=3.1623 \times 10^{-5} \mathrm{~m}$ given as functions of the mixing parameter $\Xi$ and parameterized by the span $S=S^{\mathrm{S}}=S^{\mathrm{L}}$ of the two log-normal partial distributions. The result for a monomodal log-normal size distribution with $\xi=0.3, M=3.1623 \times 10^{-5} \mathrm{~m}$ and $S=1$ is marked as D-MTM/L, 0.3 . 